\title{
MARTÍN JIMÉNEZ: UN CLÉRIGO NAVARRO ENTRE SUS OBLIGACIONES CANÓNICAS Y LA DOCENCIA UNIVERSITARIA (1268-1302)
}

\author{
POR
}

Pascual Tamburri Bariain

Universidad Pública de Navarra

\begin{abstract}
RESUMEN
La formación del clero español, al menos antes de la Reforma tridentina, tuvo como nota característica la heterogeneidad. En función de la renovación de la historia universitaria y educativa, ha de reexaminarse el vasto conjunto de estudios y materiales reunidos en las áltimas décadas. El caso de Martinus Eximinis, canonista en Italia a finales del siglo XIII, se propone aquí para plantear tanto la importancia concedida por las instituciones eclesiásticas a la formación superior de sus miembros más jóvenes y capacitados como la respuesta de éstos una vez alcanzados los grados académicos más altos en un momento de transición eclesial y de cambio social.
\end{abstract}

\begin{abstract}
The case of Martinus Eximinis, canonist in Italy around the end of $13^{\text {th }}$ century, is proposed here to outline the importance conceded by the ecclesiastic institutions to the superior education of their members more young and trained like the answer of these once reached the academic degrees higher in a moment of eclesial transition and social change.
\end{abstract}

\section{LA IGLESIA Y LOS UNIVERSITARIOS NAVARROS}

La historia de las Universidades en la Edad Media no puede hacerse sin recordar que todos los Estudios, por definición y salvo prueba en contra, siguie- 
ron siendo instituciones de la Iglesia, lo que, por una parte, otorgaba a sus miembros particulares privilegios eclesiásticos, e incluso espirituales, y, por otro, los convertía en teoría en el centro de formación superior de Aquella'. En todo caso, este carácter no tenía relación formal con la variable proporción de clérigos y laicos dentro de los cuerpos docentes y discentes, que además presentaba notables diferencias, por ejemplo, entre las trayectorias de cuatro casos modélicos como podrían ser París, Oxford, Salamanca y Bolonia. No obstante la presencia de laicos, a veces preponderante, la Iglesia nunca negó el carácter religioso de las Universidades porque siempre obtuvo de ellas los cuadros dirigentes que necesitaba para su gobierno; como ha dicho Jacques Verger, aludiendo al caso de Urbano V, en su «traicionado desengaño» Roma y toda la jerarquía mantuvieron su política de asistencia universitaria y de fundaciones (incluyendo la autorización para la concesión de grados) a sabiendas de que muchos de aquellos esfuerzos no recaerían en clérigos.

Esta situación, sustancialmente prolongada desde el siglo XII al XIX, llevó a que muchos estudiantes se ordenasen para beneficiarse de tal política, con pocas intenciones de perseverar en una vocación por lo demás inexistente. Con las investigaciones actualmente en curso, puede llegarse a definir no sólo el porcentaje de clérigos en las diferentes Facultades de las Universidades prenapoleónicas (en la línea de lo ya intentado por Sven Stelling-Michaud), sino también la proporción de éstos que realmente ejercía su ministerio y el grado y circunstancias del abandono. En esta dinámica estuvieron no sólo las Universidades más «clericales», como la de París (a-jurídica), sino también las carentes de estudios teológicos avanzados, como la prevalentemente civilista de Bolonia (que no los tuvo hasta finales del siglo XIV).

La llamada «crisis de la Teología universitaria», desde 1300, implicó también a los estudiantes y maestros españoles, y, como se ha intentado demostrar en otro lugar, llevó a una reducción de los estudiantes teólogos que, sea o no peculiar de los naturales de los reinos hispánicos, es característica de nuestra última Edad Media ${ }^{2}$. Trataremos aquí, a través de la biografía de un clérigo español en la Italia septentrional de los siglos XIII y XIV, de comprender los fundamentos de la interconexión entre las Iglesias locales y los Estudios generales, así como, ante todo, la utilidad funcional y las perspectivas sociales de la formación universitaria para un presbítero.

Pese a una voluntad reiterada, y a una necesidad en ocasiones evidente (especialmente para la Iglesia diocesana, pero también para las grandes fundacio-

1 J. VERGER, Le Università nel Medioevo, Il Mulino, Bologna, 1993, p. 119 y ss.

2 P. TAMBURRI BARIAIN, Natio hispanica. Juristas y estudiantes españoles en Bolonia antes de la fundación del Colegio de España, p. 180 (en prensa: Publicaciones del Real Colegio de España en Bolonia).

$I^{\text {er }}$ Congreso de Historia de la Iglesia

Hispania Sacra 51 (1999) 
nes regulares), Navarra jamás dispuso de un Estudio General. Los navarros, pues, nunca dejaron de acudir a las Universidades exteriores, españolas o extranjeras ${ }^{3}$. Las escuelas navarras, que no se apartaron de lo habitual en las ins.taladas en otros espacios políticos de su ámbito cultural, no sobrepasaron los primeros escalones de la enseñanza superior, y no llegaron a una definición de su autonomía en conflicto con otros poderes ${ }^{4}$ En el reino pirenaico nunca se pudo conferir la licentia ubique docendi $i^{5}$, aunque disponer de letrados, teólogos y médicos con una formación universitaria adecuada era necesario para Navarra ante todo, por las disposiciones canónicas que obligaban al clero. Así, se llegó a una solución intermedia, destinada a durar en su esencia hasta muy entrado el siglo XX, y quienes deseasen completar su preparación acudirían a los Estudios Generales ya existentes ( $\mathrm{y}$ fundamentalmente para estudios teologicos y canónicos) ${ }^{6}$.

Debe entenderse en este sentido desde el siglo XII la escuela catedralicia, mientras que los canónigos acuden a los Estudios ultrapirenaicos?. Es también el caso del clero regular y, por lo que aquí nos interesa, de Roncesvalles ${ }^{8}$. En general, se ha demostrado la preferencia continuada en los siglos medievales de los canónigos pamploneses por París y Tolosa (62 nombres conocidos) 9 . Bolonia, patria del Derecho desde el siglo XII, es el caso menos estudiado hasta el momento en su relación con Navarra; intentaremos aquí, más que tra-

3 J. GOÑ GAZTAMBIDE, «La formación intelectual de los navarros en la Edad Media (11221500)», Estudios de Edad Media de la Corona de Aragón, X, Zaragoza, 1975, p. 143 y ss.. V. DE LA FUENTE, Historia de las Universidades, colegios y demás establecimientos de enseñanza en España, Madrid, 1884 (Navarra: vol. I, p. 221 - 222), y F. ELIAS DE TEJADA, «La literatura política en la Navarra medieval», Principe de Viana, 17, Pamplona, 1956, pp. $199-212$. Un acertado estado de la cuestión: L.J. FORTÚN, La formación intelectual de los navarros en la Edad Media (siglos XII - XV), en A.J. MARTÍN DuQUE, dir., Gran Atlas de Navarra, II, Historia, Pamplona, 1986, pp. 116 - 118.

4 M.P. RÁBADE OBRadó, Las Universidades en la Edad Media, Madrid, 1996, pp. 9 - 16 y $41-42$.

5 H. RASHDALL, The Universities of Europe in the Middle Ages by the late Hastings Rashdall, dean of Carlisle. A new edition in three volumes edited by F.M. Powicke, Regius Professor of Modem History in the University of Oxford and A.B. Emden, Principal of St. Edmund Hall, Oxford, Oxford, 1936, vol. I, pp. 1 - 24, en cuanto a estos conceptos fundamentales

6. J. VERGARA CIORDIA, Colegios seculares en Pamplona (1551-1734), Estudio a la luz de sus constituciones, Pamplona, 1991, pp. 14 - 16.

7 V. BELTRÁN DE HEREDIA, «La formación intelectual del clero en España», Miscelánea Beltrán de Heredia, Salamanca, 1972, 1, p. 44. J. GONI GAZTAMBIDE, «La formación..., p. 146.

8 J. GOÑ̄ GAZTAMBIDE, «La formación..., p. 152. En general, P. TAMBURRI BARIAIN, «Presencia institucional de Roncesvalles en Bolonia (siglos XIII - XVI)», Hispania Sacra, 49, Madrid, 1997, pp. 363 - 408 (donde se recoge la documentación aquí empleada, pero el caso de Martín Jiménez es por completo marginal).

9 J. Vergara CloRdia, «La incorporación de Navarra a los saberes», en A.J. MARTín DUQUE, dir., Signos de identidad histónica para Navarra, I, Pamplona, 1996, pp. 470 - 471. J. GONi GAZTAMBIDE, «La formación..., p. 150. 
zar una perspectiva general, plantear un caso concreto, que muestra en buena medida las aspiraciones de los estudiantes de la plena Edad Media, los límites de su adscripción clerical y la flexibilida, en definitiva, del esquema canónicouniversitario.

\section{UNA FUNDACIÓN UNIVERSITARIA ESPAÑOLA}

Estando así las cosas, la presencia de navarros en Bolonia en los siglos XII, XIII y en la primera parte del XIV ha sido poco estudiada y es mal conocida, fuera de aproximaciones meramente anecdóticas o bibliográficas. La necesidad de profundizar en ese conocimiento viene impuesta por la importancia cualitativa y cuantitativa de la comunidad universitaria española en aquellas fechas, y por la relevancia de los graduados en Bolonia a su regreso a España. Ciertamente la historia de la comunidad universitaria española y de las nationes correlativas se conoce con cierto detalle después de la fundación del Colegio español, pero es preciso intentar establecer las pautas de conducta de un cierto número de canonistas navarros en Bolonia. Desde nuestro punto de vista, todos los estudiantes deberían poderse clasificar como clérigos o laicos; en la ciudad italiana, lo que falta en los documentos son datos, salvo en los contados casos en que se ha hecho alusión a este interesante aspecto. Con respecto al clero secular, la palabra «clericus» induce frecuentemente a dudas, ya que implica sólo la recepción de las órdenes menores, es decir, un estatuto administrativo peculiar. Partiendo de apreciaciones de este tipo, se ha dicho que el porcentaje de clérigos entre los estudiantes en Bolonia es mayor que en otras Universidades (el 70\% para Sven Stelling-Michaud), pero esto no parece demostrable, especialmente frente a una comunidad española compuesta por una mayoría de civilistas (aunque está lejos de probarse, incluso en el XII-XIII, la ecuación civilista $=$ laico) y con un $20 \%$ de clérigos según Antonio García y García ${ }^{10}$. Los laicos son, pues, la mayoría, en un contexto netamente clerical en el sentido más amplio, es decir, intelectual. Naturalmente, aquí cabría señalar que esta situación se transformó con la instalación del Estudio teológico, a fines del XIV, pero en poco afectó esto a los estudiantes navarros.

En efecto, la comunidad navarra en Bolonia se basó, como se ha descrito, en el hospital de Santa María de la Mascarella, destinado por la comunidad de Roncesvalles, al menos parcialmente y en momentos concretos de los siglos XIII y XIV, para sus estudiantes canonistas. Presciendiendo aquí de las otras

10 A. García Y García, «Escolares ibéricos en Bolonia, 1300-1330», en A. García y García, Derecho Común en España. Los juristas y sus obras, Murcia, 1991, pp. 21 - 22, con una ponderación razonada del porcentaje de clérigos.

$\mathrm{I}^{\mathrm{er}}$ Congreso de Historia de la Iglesia

Hispania Sacra 51 (1999) 
funciones de la institución y de su apertura al resto de españoles en Bolonia, el documento más antiguo conservado sobre la Mascarella, es del 13 de enero de $1241^{11}$. En esta fecha se determinan, con arreglo a la documentación del siglo anterior, los límites parroquiales de la Mascarella frente a la de Santa María Magdalena de S. Donato. Rector de la misma y comendador era el canónigo García de Roncesvalles, y capellán de la Mascarella el sacerdote italiano Angelo. No sabemos si el comendador era estudiante en este momento, aunque es posible, ya que en estos años hubo varios juristas españoles con el mismo nombre ${ }^{12}$. El documento nos muestra una institución en pleno funcionamiento, arraigada en la ciudad y vinculada a Roncesvalles desde tiempo atrás. Para todo lo relativo a la función pastoral en la ciudad, la Mascarella dependió siempre de los sucesivos obispos, que no dejaron de servirse de estas prerrogativas para intervenir en la vida interna de la misma. A mediados del siglo XIII, sin embargo, el mejor momento de la Mascarella no había llegado todavía. A partir de 1270 aproximadamente, con un patrimonio suficiente, fruto de compras y de donaciones, se conserva una serie bastante regular de documentos, incluyendo los nombramientos de los comendadores, ingresos y gastos, que permiten conocer mejor su funcionamiento interno. En nuestro caso, nos dan acceso a la vida de nuestro protagonista, un personaje singular en el que se retrata la trayectoria académica de una parte cualitativamente importante del clero de su siglo.

El período mejor conocido de la Mascarella se inicia en 1267, con el establecimiento por el municipio de una tasa sobre los actos jurídicos documentados, que da lugar a que queden regestos de todos los documentos de cierta importancia patrimonial (transmisiones por encima de 20 libras de valor). En 1268 se produce la compra, por parte del comendador Martín Jiménez (Martinus Eximinis), de un solar, probablemente un huerto, en el mismo barrio donde se hallaba la parroquia. Esta compra es la evidencia de una situación financiera desahogada de la encomienda, y supone la aparición del canónigo en Bolonia ${ }^{13}$. Martín Jiménez, el canónigo comendador, tuvo una larga estancia en Bolonia, que vamos a estudiar a continuación.

Antes de licenciarse en la Universidad, el 5 de noviembre de 1268, Martín Jiménez recibio 80 libras boloñesas de Preso de Boninsegna, que a cambio fue autorizado a organizar cuestaciones en nombre de Roncesvalles en la Italia

\footnotetext{
11 Bolonia, Archivo Hercolani, Rogito de Ugolino Rigazzi. 1241, елеro, 13, Bolonia.

12 M. SARTl y M. FATTORINI, De claris $I I$, p. 307 y ss.

13 F. CAlzoni, Storia della chiesa parrocchiale di Santa Maria in via Mascarella e dei luoghi (...), Bologna, 1785, p. 22.
} 
central y meridional ${ }^{14}$. Dando en arriendo esta fuente de ingresos extraordinarios, los comendadores se encontraron, al menos en los momentos más florecientes, en condiciones de financiar todas sus obligaciones e, incluso, de enviar contribuciones significativas a Roncesvalles. En un documento del 2 de enero de 1269, el mismo Martín Jiménez devuelve una finca, de la que no se precisa la superficie, al padre de una joven. Esta propiedad, que era la dote de su hija, había sido entregada a la Mascarella en previsión de que ella profesase como religiosa en el hospital boloñes; al renunciar la interesada a esa posibilidad, su padre y tutor solicitó la devolución del depósito ${ }^{15}$. El hecho en sí no es importante, pero sí es significativo como síntoma de la actividad del hospital, en el que sin duda había otras muchachas. Según F. Calzoni, existía incluso una sección femenina del hospital, o más bien dos hospitales, lo que es muy dudoso $^{16}$. Poco después, y siempre en 1269, Martín Jiménez dejó para siempre la Mascarella. A la encomienda debió lo esencial de su formación, sus excelentes relaciones en Bolonia y los comienzos de su brillante carrera docente y forense como civilista, que le proporcionó un apreciable patrimonio. Como canónigo comendador, el segundo conocido, Martín Jiménez llevó la encomienda a vivir unas décadas de esplendor material y de florecimiento intelectual (por la presencia de estudiantes), pero también a entrever los límites de ese éxito: las rentas eran inseguras, por una parte, y los estudiantes, llegados como él a estudiar cánones, preferían el derecho civil, más rentable para ellos, pero ruinoso a la larga para la inversión que en ellos hacía Roncesvalles. El 13 de mayo de 1269 Domingo García, otro canónigo del hospital pirenaico, sucedió a Martín Jiménez en la encomienda ${ }^{17}$.

\section{IDENTIFICACIÓN DEL PERSONAJE}

Vemos, pues, que entre 1268 y 1302 un cierto Martinus Hispanus aparece con frecuencia en la documentación boloñesa. Su actividad en el Estudio y en la ciudad ha sido objeto de algunas menciones en la bibliografía al uso, pero su biografía no ha sido todavía escrita. La vida de este clérigo español, maestro en Bolonia, sirve para marcar la transición entre dos siglos radicalmente diferentes por lo que se refiere a la presencia hispana en el Alma Mater.

\footnotetext{
14 Bolonia, Archivio di Stato [A.S.B.], Memoriali, Amadore della Croara.1268, noviembre, 5, Bolonia.

15 A.S.B., Memoriali, Semprebene del Nero. 1269, enero, 2, Bolonia.

16 F. CALZONI, Storia, p. 18.

17 A.S.B., Memoriali, Rolandino Bonsignori. 1269, mayo, 13, Bolonia.

$\mathrm{I}^{\text {er }}$ Congreso de Historia de la Iglesia

Hispania Sacra 51 (1999)
} 
En efecto, entre los primeros documentos de los notariales boloñeses conservador en la serie de los Memoriali se encuentran varios tipos distintos de referencias a este personaje: a) Las relativas a Martinus Eximini Yspanus, comendador de la iglesia y hospital de Santa María de la Mascarella en 1268 69; b) las de Martinus Eximini, de Teruel (o más bien beneficiado de Teruel), en 1276 y 1282; c) las del maestro canonista Martinus Eximini Yspanus en 1281 - 82; d) las de Martinus Egidii de Seminis, rector ecclesie de Cuba, cesaraugustensis diocesis, en 1282; y, en fin, e) Martinus Hispanus, profesor de lógica en $1275^{18}$. Las menciones de los cuatro primeros tipos pueden identificarse sin problemas con un único universitario español, clérigo de Roncesvalles que realizó estudios completos siendo comendador en Italia y que tras licenciarse (en ambos derechos, y no sólo en cánones) permaneció en Bolonia como maestro; las menciones del quinto tipo presentan más dudas, pudiendo tratarse de un homónimo. El canónigo Martín Jiménez está, pues, en Bolonia como estudiante de leyes desde al menos 1268 , pero su trayectoria académica no fue en absoluto ordinaria.

Sobre la primera parte de su biografía, sin embargo, ya hemos dicho cuanto aquí conviene. Llegado al Estudio gracias a la Mascarella, la dirigió en un momento de esplendor pero la abandonó para desarrollar su propia carrera en los años finales del siglo XIII, cuando en Bolonia coinciden algunos maestros españoles con el inicio del auge numérico de los estudiantes ${ }^{19}$. Sólo tenemos la certeza de que está en la Mascarella hasta el 2 de enero de 1269, con el hospital funcionando plenamente, como ya se ha visto ${ }^{20}$. Después, y sin que conste un enfrentamiento con Roncesvalles, Martín se independizó y fue sustituído por el comendador Domingo García, aunque consta que siguió siendo escolar cuando menos hasta el 11 de agosto de 1274; el 21 de diciembre de 1282 parece ser ya miembro del colegio de doctores, y de hecho en 1286 patrocino un candidato para la entrada en el colegio de canonistas, lo que implica que debía

\footnotetext{
t8 Respectivamente a) véanse notas 14 y 15 , supra, b) M. SARTI y M. FATTORINI, De claris Archigymnasi Bononiensis Professoribus a saeculo XI usque ad saeculum XIV. Iterum ediderunt Caesar Albicinius () et Carolus Malagola. Tomus II, Bononiae, 1896, p. 315 y 321; c) M. SARTI y M. FATTORINI, De claris II, pp. 320 y 321. A. GARCÍA y GARCíA, Escolares, pp. 39 - 40, n. 66 y 67 (sugiriendo la identidad entre ambos personajes); d) M. SARTI y M. FATTORINl, De claris II, p. 321; Egidii parece una lectura errónea de Eximini; e) M. SART1 y M. FATTORINl, De claris Archigymnasi Bononiensis Professoribus a saeculo XI usque ad saeculum XIV. Iterum ediderunt Caesar Albicinius () et Carolus Malagola. Tomus I, 1888, p. 593.

19 P. TAMburrl BarialN, «Presencia..., p. 381 y ss. En general, F. CAlZONI, Storia, p. 22. P. RODRÍGUEZ-PONGA, en un meritorio trabajo (inédito) de 1935, recordaba un "Martinus Hispanus», aragonés, que sin duda se identifica con éste.

20 M. SARTI y M. FATTORINi, De claris, $I$, p. 315, en 1276 , p. 320, en 1281 , p. 21 , en 1282 , y, como maestro, M. SARTI y M. FATTORINI, De claris $I$, p. 483.
} 
ser ya un miembro prominente del mismo ${ }^{21}$. Hay que suponer que, con ciertas perspectivas profesionales abiertas en Italia, renunció en 1269 a su dependencia de Roncesvalles y a la gestión de la Mascarella; doctor en ambos Derechos, nunca volvió a España.

\section{VIDA PROFESIONAL}

Como maestro canonista y jurisperito civilista, su prestigio en Bolonia es incontrovertible, como prueba la embajada que la ciudad le encargó en 1294, junto al civilista Gardino y a su colega canonista Bonincontro, en la corte del conde de Romagna Hildebrandino. El 27 de septiembre de 1297, en los días no lectivos entre los dos años académicos, Martín Jiménez fue encargado por las Universidades ( $«$ sindicus et procurator rectorum et universitatis scholarium ultramontanorum et citramontanorum») de percibir 150 libras en préstamo de los banqueros florentinos della Scala, para pagar con ellas el salario del canonista de Narbona Stephanus Boverii ${ }^{22}$. En este momento de máxima gloria académica y política, el 1 de julio de 1298 , fue testigo del nombramiento del arcediano de Bolonia Guido di Baysio. Con estos pocos datos, Martín Jiménez deja de sí una imagen de maestro ultramontano perfectamente insertado en la sociedad civil boloñesa. Como canonista, no produjo una obra relevante y conocida, pero en cambio tuvo una considerable práctica docente, política y probablemente forense, que le reportaron excelentes contactos en la ciudad y una fortuna personal no desdeñable. Como contrapartida, su italianización fue total, y Martín Jiménez, en el momento de su muerte, había cortado todos los vínculos con la comunidad española, que no aparece representada en la compleja documentación de su testamentaría. Consciente de su decisión de quedarse para siempre en Bolonia, él se hizo en todo italiano, y, a diferencia de otros célebres maestros españoles, más activos científicamente pero menos partícipes de la vida boloñesa, no volvió a España ni siquiera a morir.

El «caso» Martín Jiménez indudablemente preocupó a Roncesvalles, tanto por los posibles riesgos que una repetición de su abandono supondría para el

21 M. SARTl y M. FATTORINI, De claris I, p. 483 - 484; . A.L. TROMBETTl, L'esame di laurea presso lo Studio bolognese. Laureati in diritto civile nel secolo XV, en BRIZZI, G.P., y PINI, A.I., dir., Studenti $e$ università degli studenti a Bologna dal XII al XIX secolo, "Studi e memorie per la storia dell'Università di Bologna», n.s., VII, Istituto per la storia dell'Università, Bologna, 1988, p. 147 y ss., además de G. CENCETT1, La laurea nelle Universita Medievali, «Studi e Memorie per la storia dell'Università di Bologna», 16, Bologna, 1943, p. 249 - 273, y L. PAOLINI La laurea medievale, en O. CAPITANI, dir., L'Università a Bologna. Personaggi, momenti e luoghi dalle origini al XVI secolo, Cassa di Risparmio in Bologna, Milano, 1987. pp. 133 - 156.

22 M. SARTI y M. FATTORINI, De claris I, p. 484.

$\mathrm{I}^{\mathrm{er}}$ Congreso de Historia de la Iglesia Hispania Sacra 51 (1999) 
patrimonio de la Mascarella (un comendador dimisionario con menos escrúpulos podría enriquecerse con gran facilidad) como por la difícil selección de los canónigos a los que dar en Bolonia una formación superior, dado el riesgo de perder, atraídos por las nuevas posibilidades profesionales, a los canónigos más capaces intelectualmente. La respuesta fue Pedro Jiménez. Canónigo de Roncesvalles y comendador de Santa María de la Mascarella desde el 13 de diciembre de $1275^{23}$, tuvo, junto a las responsabilidades administrativas de sus predecesores, también la dirección espiritual de la parroquia, sustituyendo a los precedentes capellanes. Este cambio marca el momento de máxima dependencia de Roncesvalles. En efecto, no sólo la dirección seguía siendo navarra, y también presumiblemente, como veremos, una parte de los beneficiarios de la asistencia hospitalaria, sino que también la parroquia fue gestionada directamente. En 1269 y 1270 nos encontramos con un Pedro Jiménez, capellán «de Burdeos», estudiante hispano de leyes ${ }^{24}$. Todo hace pensar, pues, que el comendador residía ya en la Mascarella desde hacía algún tiempo en el momento de su nombramiento, como era tal vez habitual, y conviviría en este caso con Martín Jiménez. Su gestión se prolongó hasta 1308, con una estancia en Italia tan larga como la de nuestro biografiado, pero mucho más útil para Roncesvalles.

\section{LA HERENCIA DE MARTÍN JIMÉNEZ}

Once documentos en los Memoriali de 1300 se refieren a Martinus Hispanus y a sus bienes. Sabemos que el maestro murió en Bolonia o su comarca a lo largo de la primera mitad del año, porque el 12 de mayo vivía todavía, en las proximidades de $S$. Andrea degli Ansaldi; en esa fecha compró un solar en Borgo Panigale a Lodomintus Johannis de Cento, por 59 libras, tres sueldos y cuatro dineros. Todavía el 20 de junio vendio a un cierto Vandinus diversos terrenos por 607 libras y 7 sueldos. Cifras, como vemos, bastante considerables $^{25}$, en un momento inmediatamente anterior a la muerte del jurista. En efecto, aunque no sabemos la fecha ni el lugar exactos, y no hay rastro de su testamento en la documentación notarial, todo lleva a pensar que falleció cerca de Bolonia pero fuera de la ciudad propiamente dicha (en las poblaciones don-

23 Bolonia, Archivo Arzobispal, Libro delle Asse del Capítulo catedralicio de S. Pedro: 1275, di ciembre, 13, Bolonia.

24 M. SART! y M. FATTORINI, De claris, II, p. 310 y 312.

25 L. CONTINELLI, L'archivio dell'ufficio dei memoriali. Inventario, I, I Memoriali, 1265 - 1436 Tomo I, 1265 - 1333, Istituto per la storia dell'Università, Bologna, 1988, pp. 71 - 74: volúmenes 98 (primer semestre), 99 (primer y segundo semestre) y 100 (parcialmente, segundo semestre).A.S.B. Memoriali, 098, 1300, fol. 242r. [243r.], 4 (1. 25-29), y A.S.B., Memoriali, 098, 1300, fol. 125r.$125 v ., 3(1.46-58$ y $1-26)$. 
de se situaba buena parte de sus intereses). Sin rastro de su testamento, tal vez porque no fuese debidamente registrado, ya el 25 de julio ${ }^{26}$ Paganus de Caxate, milanés, nombró procuradores en Bolonia (Francesco de Medios de Milán y el dominicano Gaspar) para recuperar un crédito de cien libras de los herederos de Martín por deudas de éste. Considerando que la noticia del fallecimiento debería haber llegado a Lombardía al menos una semana antes, Martín Jiménez pudo morir entre el 20 de junio y tal vez el 15 de julio de 1300 . Si había llegado a Bolonia entre los 20 y los 30 , y se había doctorado más o menos cinco años después, el canonista tendría entre 55 y 65 años al morir.

Cinco días después, el 30 de julio, aparece por primera vez el heredero de Martín. Bonifacio de Langlano, en tal condición, pagó en esa fecha 89 libras a Francesco Allamanni de' Guarinis por una pequeña viña de algo más de una hectárea en Borgo Panigale que el maestro había comprado poco antes de morir y no había pagado todavía. Esto nos permite afirmar que su muerte había sido totalmente inesperada, mientras estaba todavía consolidando su situación patrimonial en los inmediatos alrededores de la ciudad, a partir de un sólido prestigio forense y docente. De Bonifacio, el heredero lombardo, no sabemos casi nada; raramente estaba en Bolonia atendiendo sus intereses, y así, por ejemplo, el 29 de agosto, Martino Thomaxini de Marsiliis era su procurador en la ciudad, representado a su vez por fray Rodolfo, de la Orden de San Juan de Jerusalén. Al día siguiente, 30 de agosto, este fray Rodolfo, el dominico Giovanni da Faenza y Martino de Marsiliis, como procuradores de Bonifacio, venden la viña de Borgo Panigale comprada un mes antes. En este documento, aunque se confirma el origen milanés de Bonifacio, se explica su vinculación con Bolonia, y por consiguiente con el español Martín, al llamársele «canonicus bononiensis» ${ }^{27}$.

El 6 de septiembre, como saldo parcial de la parte de la herencia (bienes o créditos) situada fuera de la ciudad y de su territorio, Bonifacio recibió 114 libras del banquero Gomes Zantis de Pistoia, ejecutor del testamento en aquella ciudad. El 7 de octubre, el ya citado Paganus de Caxate (a través de sus procuradores fray Gaspar y Francesco de Medios) recibieron 100 libras del testamento de Martín, tal vez por disponer los ejecutores de suficiente liquidez en aquel momento, gracias a la remesa del mes anterior. El 27 de noviembre, Bonifacio vendió una finca en Borgo Panigale a Rolandino Lamberti Bonçagnis por 374 libras; la mayor parte de los bienes inmuebles de Martín Jiménez se concentran en una zona muy concreta en el Noroeste de Bolonia. El mismo

26 A.S.B., Memoriali, 099, 1300, fol. 187v., 3 (1. 18-23).

27 A.S.B., Memoriali, 099, 1300, fol. 269r., 1 (1. 3-13), A.S.B., Menoriali, 099, 1300, fol. 351v, 3 (1. 35-43), y A.S.B., Memoriali, 099, 1300, fol. 282r., 2 (1. 20-30). La viña era de unas cinco «tomature», $\mathrm{y}$ fue vendida por 110 libras a un cierto Bonundo, que evidentemente no hizo un buen negocio.

$I^{\text {er }}$ Congreso de Historia de la Iglesia Hispania Sacra 51 (1999) 
día, nombró otros dos procuradores -Paxanus de Altedo y el doctor Conadoris de' Guidonis - para cobrar algunas deudas impagadas de Martín en Bolonia. Tres días después, a través de Horiaus pagó a los tres anteriores procuradores 60 libras que Bonifacio les debía por gastos hechos en la gestión de la herencia del español ${ }^{28}$. Sin embargo, el complejo proceso de asunción y liquidación del patrimonio (razonablemente extenso pero no enorme) no había terminado.

El 19 de agosto de 1301, en efecto, Bonifacio pagó a Borghexanus Ugolini 200 libras que Martín Jiménez le debía. El 29 del mismo mes Bonifacio de Loglano, esta vez sirviéndose de los procuradores habituales, recibió 90 libras que Liurgungo [?] Alberti y Jacobus de Placi debían a Martinus Ispanus. El 12 de octubre, Bonifacio vendió una casa en el mismo Borgo Panigale, con un terreno menor de una hectárea, heredada del maestro Martín, a Prone [?] Pellegrini Symoni por 89 libras. En cambio, el 2 de noviembre (mediante el procurador Amadore Albertini) tuvo que pagar una deuda, del mismo origen, de 40 florines, a Anselmo de Bérgamo. Finalmente, Bonifacio consiguí liquidar la herencia en 1302. El 5 de abril, Venturino Bresani de Valleriis recibió de un procurador del milanés Bonifacio de Langlano -Brabanaxius de Azoguidis30 libras boloñesas que le debía Martinus Ispanus ${ }^{29}$.

\section{CONCLUSIONES}

La muerte de Martín Jiménez, aparentemente súbita, dejo su obra patrimonial sin terminar, al menos en relación a las expectativas que su 6ptima inserción social en Bolonia le permitía sin duda mantener. Su patrimonio fundiario, sin ser enorme, podría haberse convertido en la garantía de una vejez tranquila para un clérigo jurista español que llevaba más de treinta años viviendo como un boloñés. De su heredero, el italiano Bonifacio, no se conoce nada, ni siquiera la relación que tuvo con el causante. Puede pensarse que se trató de un discípulo del español, elegido como heredero por una persona sin vínculos familiares en su patria de origen. Por lo que dejó (y, al faltamos el testamento, no sabemos nada de sus bienes muebles y especialmente de sus libros), Martín

28 A.S.B., Memoriali, 100, 1300, fol. 313v., 5 (1. 47-59), A.S.B., Memoriali, 099, 1300, fol. 223v., 1 (1. 9-16), A.S.B., Memoriali, 100, 1300, fol. 89r.-89v., 2 (1. 63-70) y 2v, (l. 1-18), A.S.B., Memoriali, 100, 1300, fol. 89v., 1 (1. 19-26) y A.S.B., Memoriali, 099, 1300, fol. 311 v., 2 (l. 13-30).

${ }^{29}$ L. ContinelLi, L'archivio, p. 74 - 76; Volúmenes 100 (parcialmente, segundo semestre), 101 (primer semestre) y 102 (segundo semestre), A.S.B., Mernoriali, 102, 1301, fol. 383r.[386r.], 4, (1. 2134), .AS.B., Memoriali, 102, 1301, fol. 86v., 1 (1. 7-16)., A.S.B., Memoriali, 102, 1301, fol. 109r., 3 (1. 20-36) y A.S.B., Memoriali, 102, 1301, fol. 426r., 6 (1. 36-42). 1302, L. CoNTINELLI, L'archivio, p. 76 - 79: Volúmenes 103 (primer semestre), 104 (segundo semestre) y 105 (segundo semestre). A.S.B., Memoriali, 103, 1302, fol. 170r. (1. 39-46). 
Jiménez parece haber tenido una vida profesional bastante fructífera; sin duda no era el porvenir que le reservaban sus superiores de Roncesvalles cuando lo enviaron a la Mascarella, pero, quedándose en Italia, había obtenido una posición muy respetable en la ciudad, como punto de partida de un siglo que vio, sucesivamente, el final de la presencia significativa de maestros españoles, el auge y declive de la comunidad estudiantil y, fuera del período que a continuación nos ocupará, su definitiva consolidación con el Colegio de España.

Junto a estos maestros y precursores, la dimensión universitaria de la comunidad regular de Santa María de Roncesvalles en Bolonia ${ }^{30}$, nunca negada, necesita un mejor conocimiento. La encomienda de Santa María 'de la Mascarella' en la ciudad universitaria resulta ser, más que una inversión económicamente productiva, una inversión en la preparación universitaria de los canonigos y, en ocasiones, el punto de agregación de toda la comunidad hispana en la ciudad. La formación boloñesa de sus estudiantes - con los riesgos de todo tipo que Martín Jiménez encarnó- situó por un tiempo a Roncesvalles y a Navarra a la cabeza de los reinos peninsulares en este sentido, hasta que la decadencia de la pequeña encomienda dio claramente paso a la preferencia por las facultades francesas.

Según los Estatutos de Roncesvalles, un comendador, no necesariamente canónigo, habría de establecese en Bolonia, con competencias sobre toda Italia y amplia libertad para reclutar personal y gestionar el patrimonio: unas normas en las que es evidente el peso de la experiencia, aunque no siempre los comendadores respondieron a las expectativas. Lo único cierto sobre el arranque de la presencia de Roncesvalles en Italia es que nunca llegaremos a conocer la fecha exacta. Los objetivos que el cabildo de Roncesvalles tuviese al fundar y/o mantener una encomienda en Bolonia marcan decisivamente el carácter de la Mascarella y pueden servir para explicar algunos aspectos peor conocidos de la historia de ésta. En primer término, como todas las propiedades, encomiendas, derechos y fundaciones relacionados con Roncesvalles, el destino de la Mascarella era la rentabilidad, cifrada, en primer lugar, en la autosuficiencia, y después en sus remesas a la comunidad titular. Por otra parte, la Mascarella siempre mantuvo un hospital, y esta actividad, bajo una u otra forma, duró más que la propia dependencia de la institución española. En tercer lugar, Santa María de la Mascarella tuvo una función universitaria que explica y complementa las dos anteriores. Casi desde su fundación, la Mascarella recibió limosnas y donativos para ayudar a los pauperes extranjeros venidos a la ciudad; ahora bien, no se trata de una común actividad asistencial, sino de una específica actividad universitaria, ya que, en la Bolonia del siglo XIV, y con

30 P. TAmburri Bariain, «Presencia..., p. 374 y ss..

re Congreso de Historia de la Iglesia Hispanja Sacra 51 (1999) 
más razón en el anterior, los extranjeros pobres eran por definición los escolares ultramontanos. En esto, la Mascarella parece tener una amplia autonomía operativa de Roncesvalles, ya que recibe directamente los donativos, y un programa de ayuda que se basa en alojar, educar y someter a disciplina a los estudiantes. Si se observan los desórdenes de la vida de los estudiantes en los primeros siglos de Universidad (y también después), no puede sorprender el programa universitario del hospital. Incluso cuando Roncesvalles perdio la gestión de la Mascarella, conservó el detecho de mantener allí alojado algún canónigo, facultad a la que el Cabildo se aferró tenazmente. Roncesvalles nunca tuvo una escuela propia para formar teólogos y canonistas, pero, al enviar sistemáticamente a la Mascarella a sus canónigos, con frecuencia como comendadores, podía acceder de manera privilegiada a estudios entre los más prestigiosos. El siglo XIII fue de verdadero despegue organizativo y patrimonial, y en él se dio la mayor presencia de estudiantes navarros.

Martín Jiménez fue, en conclusión, un canónigo de Roncesvalles que en 1269 terminó sus estudios y, en lugar de volver a Navarra, se estableció en Bolonia como profesor de Derecho civil y jurisconsulto, abandonando por lo que sabemos toda relación con Ia Mascarella y con Roncesvalles. Su carrera universitaria y su patrimonio personal son excepcionales en la historia de la Mascarella; seguramente fue enviado a Italia para que completase su formación canónica pero, al llegar la hora del retorno, prefirió asentarse en la ciudad como clérigo secular antes que volver al Pirineo. No abandono, pues, su ministerio, sino que puso a su propio servicio, y no al de Roncesvalles, los conocimientos adquiridos. Su vida en Bolonia nada tuvo que ver después con los avatares de la institución navarra ni con la trayectoria de la comunidad estudiantil española y al morir, en 1300 , ni sus herederos ni los testigos de su testamento fueron españoles. Italianizado, Martín Jiménez es una muestra notable del papel de la Iglesia, a través de la formación superior del clero, como mecanismo de ascenso social. Naturalmente, no sólo en la Edad Media, las comunidades regulares han aceptado un cierto porcentaje de defecciones en atención al bien mayor conseguido con los que perseveran. 